\title{
Effects of tocainide on left ventricular performance at rest and during acute alterations in heart rate and systemic arterial pressure An echocardiographic study ${ }^{1}$
}

\author{
WILLIAM F. RYAN ${ }^{2}$ AND JOEL S. KARLINER \\ From the Cardiology Division, Department of Medicine, University of California Medical Center, \\ San Diego, California, USA
}

SUMMARY To determine if the new antiarrhythmic agent tocainide depresses left ventricular function we performed a double-blind cross-over trial of tocainide and placebo in 10 patients with valvular heart disease and recorded echocardiograms (a) in the basal state, (b) during atropine-induced increases in heart rate, and (c) during acute pressure loading with phenylephrine. The drug doses were $400 \mathrm{mg}$ every 8 hours for 3 days, and the mean tocainide plasma concentration in the tocainide group was $5.3 \mu \mathrm{g} / \mathrm{ml}$.

Except after atropine when there was a slightly higher mean systolic blood pressure in the tocainide group than in the placebo group (mean blood pressure difference $7 \mathrm{mmHg}, \mathrm{P}<0.02$ ) heart rates and systolic blood pressures for both groups were closely matched during these pharmacological interventions. For the placebo and tocainide groups mean heart rates increased after atropine by 27 and 26 beats/minute, respectively, and during infusion of phenylephrine (when no significant heart rate slowing occurred) mean blood pressures rose by $51 \mathrm{mmHg}$ in the placebo group (to $175 \pm 6 \mathrm{mmHg}$ ) and by $45 \mathrm{mmHg}$ in the tocainide group (to $176 \pm 6 \mathrm{mmHg}$ ). During tocainide administration mean velocity of circumferential fibre shortening (mean VCF) in the basal state averaged $1.29 \pm 0.06 \mathrm{diam} / \mathrm{s}$. This increased to $1.39 \pm 0.08 \mathrm{diam} / \mathrm{s}$ with atropine (NS) and then declined to $1.17 \pm 0.09 \mathrm{diam} / \mathrm{s}$ during infusion of phenylephrine $(P<0.01$ vs atropine). No significant differences were found when the corresponding values during placebo were compared with these values. Similar directional changes occurred in the average mean posterior wall velocity (mean VPW) during tocainide administration when the corresponding values were $0.75 \pm 0.05,0.87 \pm 0.07(\mathrm{P}<0.05)$, and $0.71 \pm 0.05(\mathrm{P}<0.01$ vs atropine $) \mathrm{s}^{-1}$, respectively. Again placebo values did not differ significantly from tocainide values.

Thus, in patients with valvular heart disease tocainide in the dose employed has no significant depressant effect on left ventricular function either in the basal state or during acute alterations in heart rate or blood pressure. These data suggest that tocainide can be used safely for antiarrhythmic therapy in patients with mild to moderate left ventricular dysfunction.

Recent reports of animal (Duce et al., 1973; Coltart et al., 1974) and human studies (McDevitt et al., 1976; Winkle et al., 1976; Woosley et al., 1977; Ryan et al., 1978) indicate that an oral analogue of lignocaine, tocainide, is effective in suppressing ventricular arrhythmias and is a promising new antiarrhythmic agent. However, high doses of tocainide

\footnotetext{
1This study was supported in part by the Specialized Centre of Research on Ischaemic Heart Disease, NIH Research Grant awarded by the National Heart, Lung and Blood Institute, and by a grant from the Astra Pharmaceutical Corporation.

'Present address: St Vincent's Hospital, Melbourne, Australia. Received for publication 16 June 1978
}

in anaesthetised dogs have been shown to depress left ventricular function (Coltart et al., 1974). To determine whether tocainide similarly depresses myocardial function in man we administered typical therapeutic doses of the drug to patients with organic heart disease, and using ultrasound assessed left ventricular function at rest and during acute alterations in heart rate and systemic arterial pressure.

\section{Patients and methods}

Ten patients without clinical evidence of coronary artery disease but with known heart disease formed 
Table 1 Clinical data

\begin{tabular}{|c|c|c|c|c|}
\hline $\begin{array}{l}\text { Case } \\
\text { no. }\end{array}$ & $\begin{array}{l}\text { Age } \\
\text { (y) and } \\
\text { sex }\end{array}$ & Diagnosis & $\begin{array}{l}\text { Functional } \\
\text { class (NYHA } \\
\text { classification) }\end{array}$ & Treatment \\
\hline 1 & $52 \mathrm{~F}$ & MR & II & Digoxin \\
\hline 2 & $40 M$ & $\begin{array}{l}\text { Prolapsing } \\
\text { mitral leaflet }\end{array}$ & I & - \\
\hline 3 & $32 \mathrm{~F}$ & $\begin{array}{l}\text { Prosthetic } \\
\text { aortic valve }\end{array}$ & II & $\begin{array}{l}\text { Warfarin, oral } \\
\text { contraceptives }\end{array}$ \\
\hline 4 & $62 \mathrm{M}$ & AR, mild $M R$ & II & Digoxin \\
\hline 5 & $43 F$ & MR, MS & II & $\begin{array}{l}\text { Digoxin, } \\
\text { propranolol }\end{array}$ \\
\hline 6 & $32 \mathrm{~F}$ & AS, AR & I & - $^{-10}$ \\
\hline 7 & $54 \mathrm{~F}$ & MR & I & - \\
\hline 8 & $68 \mathrm{M}$ & $\mathbf{A R}$ & II & $\begin{array}{l}\text { Triamterene, hy- } \\
\text { drochlorothiaz- } \\
\text { ide, glyceryl } \\
\text { trinitrate }\end{array}$ \\
\hline 9 & $24 \mathrm{M}$ & PS & I & - \\
\hline 10 & $23 M$ & $\mathrm{AR}$ & I & - \\
\hline
\end{tabular}

MS, mitral stenosis; MR, mitral regurgitation; AS, aortic stenosis; AR, aortic regurgitation; PS, pulmonary stenosis.

the study group (Table 1). Their ages ranged from 23 to 68 years, with a mean of 43 years. All were in sinus rhythm. Control echocardiograms were first performed to familiarise the patients with the procedure, the personnel, and the surroundings. Each patient then underwent two echocardiographic studies one week apart. One study followed treatment with tocainide, the other treatment with placebo, and the order was double-blind. The drugs were administered in a dose of $\mathbf{4 0 0} \mathrm{mg}$ every 8 hours for the 3 days before each study and all routine medications were continued. The code was not broken until the study was completed and the echocardiograms had been analysed.

An identical procedure was followed during both studies. Initial inquiry was made regarding possible drug-related sensitivity, a 12-lead electrocardiogram was recorded, urine was collected for analysis, an intravenous infusion of 5 per cent dextrose and water was begun, and a blood sample was obtained to evaluate haematological, hepatic, and renal function as well as plasma tocainide concentration and, thus, patient compliance. When the pulse rate and blood pressure (cuff sphygmomanometer) were stable for at least 30 minutes an echocardiogram was recorded. Subsequently atropine was administered intravenously in a quantity sufficient to raise the heart rate approximately 30 beats/minute or until $1.5 \mathrm{mg}$ had been given. When the heart rate and systemic arterial pressure were stable for at least 5 minutes another echocardiogram was obtained. As previously described (Hirshleifer et al., 1975; Crawford et al., 1976), a solution of phenylephrine $(40 \mathrm{mg} / \mathrm{l})$ was then infused over 5 to 10 minutes in a quantity sufficient to raise the systolic arterial pressure approximately 40 to $50 \mathrm{mmHg}$.
With the heart rate response to increased arterial pressure blocked by atropine a final echocardiogram was obtained. The infusion was then terminated. The total volume of fluid used in a study was never more than $70 \mathrm{ml}$. During the second study a deliberate attempt was made to match the heart rate and blood pressure responses with those of the first study.

All patients gave informed written consent to the study procedure which was approved by the Committee on Investigations/Activities Involving Human Subjects, University of California, San Diego. Three of the 10 patients experienced mild adverse reactions while taking tocainide. These symptoms consisted of nausea in one, dizziness in one, and a brief cold sweating episode in one. Two additional patients with withdrawn from the study because of more severe side effects caused by tocainide. One experienced severe nausea and vomiting, and the other rigors. No patient had side effects while taking placebo.

Patients with coronary artery disease or congestive cardiomyopathy were not included in the study because of the concern that raising their heart rates and systemic arterial pressures might be deleterious. We recognise that the choice of patients with valvular heart disease, especially those with mitral and aortic regurgitation, could have affected our results, especially since increases in heart rate and blood pressure may have opposing effects on the beat-to-beat magnitude of regurgitation. Nevertheless, the statistical method we employed, that is, using each patient as his or her own control during the repetition of an identical procedure, should exclude any bias in the results due solely to changes in haemodynamics.

Echocardiograms were recorded with a Picker ultrasonoscope using a $2.5 \mathrm{MHz}$ transducer focused at $7.5 \mathrm{~cm}$. Recordings were made on a Honeywell Visicorder, model 1856, at a paper speed of $100 \mathrm{~mm} / \mathrm{s}$. An electrocardiogram and an indirect carotid arterial pulse tracing were recorded simultaneously with the echocardiogram. The patients were studied in the partial left lateral decubitus position. The 'standard intercostal space' technique was employed to obtain reproducible echocardiograms of the endocardial surface of the left ventricle at the level of the chordae tendineae (Popp et al., 1975). Left ventricular dimensions were measured between the endocardial surfaces of the posterior wall and the left side of the septum (Fig. A). The end-diastolic dimension (EDD) was measured at the peak of the $R$ wave of the electrocardiogram. The end-systolic dimension (ESD) was measured before the dicrotic notch on the carotid arterial pulse tracing at the point on the echocardiogram where 
A B

C

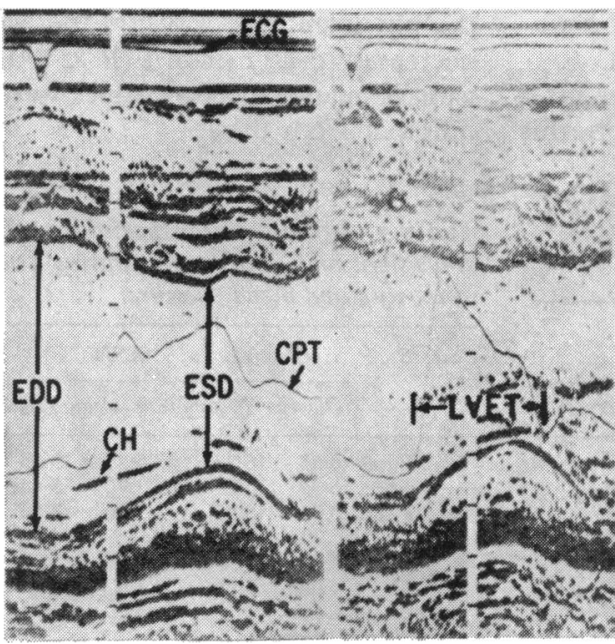

BASAL

ATROPINE

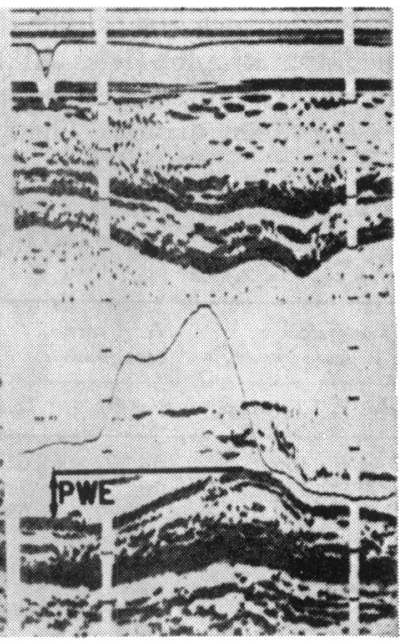

PHENYLEPHRINE

Fig. Echocardiographic recordings during tocainide therapy. (A) Basal state echocardiogram. For the beat illustrated values were: mean VCF, 1.20 diam $/ \mathrm{s}$; mean VPW, $0.75 \mathrm{~s}^{-1}$; left ventricular ejection time $321 \mathrm{~ms}$. The heart rate was 65 beats/min. EDD, end-diastolic dimension; ESD, end-systolic dimension; $C H$, chordae tendineae; CPT, carotid arterial pulse tracing; ECG, electrocardiogram. (B) After administration of atropine the heart rate increased to 95 beats/min, mean $V C F$ increased to 1.31 diam $/ \mathrm{s}$, and mean $V P W$ increased to $0.88 \mathrm{~s}^{-1}$. Left ventricular ejection time (LVET) decreased to $261 \mathrm{~ms}$. (C) During phenylephrine administration the systolic arterial pressure increased by $60 \mathrm{mmHg}$, and the heart rate fell to 85 beats/min. Mean VCF decreased to 0.95 diam $/ s$, mean VPW decreased to $0.49 \mathrm{~s}^{-1}$, and LVET increased to $340 \mathrm{~ms}$. PWE, posterior wall excursion during systole.

the perpendicular distance between the endocardial surfaces was smallest. The left ventricular ejection time (LVET) was measured from the indirect carotid arterial pulse tracing (Fig. B).

The mean normalised velocity of circumferential fibre shortening (mean VCF [diam/s]) was calculated as:

$$
\frac{\text { EDD }- \text { ESD }}{\text { EDD } \times \text { LVET }}
$$

The mean normalised posterior wall velocity (mean VPW $\left[\mathbf{s}^{-1}\right]$ ) was calculated as:

$$
\frac{\text { PWE }}{\text { EDD } \times \text { LVET }}
$$

where PWE = total excursion of the posterior wall endocardium during systole (Fig. C). All calculations were made using the average of at least 3 cardiac cycles. For data reduction a repeated measures analysis of variance was used (Winer, 1971).

\section{Results}

Individual patient data are detailed in Tables 2-4. During tocainide the mean heart rate in the basal state was $61 \pm 2$ (SE) beats $/ \mathrm{min}$. This increased to $87 \pm 6$ beats $/ \mathrm{min}$ after atropine $(P<0.01)$. During phenylephrine infusion the mean heart rate fell slightly to $81 \pm 5$ beats/min (NS vs atropine). Mean heart rates during placebo administration were not significantly different from these values.

The mean systolic arterial pressure in the basal state was $123 \pm 6 \mathrm{mmHg}$ during placebo and $131 \pm 5 \mathrm{mmHg}$ during tocainide $(P<0.01)$. After atropine these values were $124 \pm 6 \mathrm{mmHg}$ (placebo) and $131 \pm 5 \mathrm{mmHg}$ (tocainide) $(P<0.02)$. These values were not significantly different from the respective values in the basal state. During infusion of phenylephrine the mean systolic blood pressures for the placebo and tocainide groups were $175 \pm$ $6 \mathrm{mmHg}$ and $176 \pm 6 \mathrm{mmHg}$, respectively (NS). The phenylephrine-induced increases in systolic 
blood pressure, which averaged $51 \mathrm{mmHg}$ (range 28 to $88 \mathrm{mmHg}$ ) during placebo and $45 \mathrm{mmHg}$ (range 32 to $79 \mathrm{mmHg}$ ) during tocainide, differed significantly from values after atropine $(P<0.01)$.

The average mean VCF in the basal state after tocainide was $1.29 \pm 0.06 \mathrm{diam} / \mathrm{s}$. Though this

Table 2 Effects of oral tocainide on blood pressure and heart rate in basal state and after atropine and phenylephrine

\begin{tabular}{|c|c|c|c|c|c|}
\hline \multirow[t]{2}{*}{$\begin{array}{l}\text { Case } \\
\text { no. }\end{array}$} & \multirow[t]{2}{*}{ State } & \multicolumn{2}{|c|}{$\begin{array}{l}\text { Systemic systolic } \\
\text { blood pressure }(\mathrm{mmHg})\end{array}$} & \multicolumn{2}{|c|}{$\begin{array}{l}\text { Heart rate } \\
\text { (beats } / \text { min) }\end{array}$} \\
\hline & & Placebo & Tocainide & Placebo & Tocainide \\
\hline 1 & $\begin{array}{l}\text { Basal } \\
\text { At } \\
\text { Ph }\end{array}$ & $\begin{array}{l}158 \\
160 \\
194\end{array}$ & $\begin{array}{l}156 \\
158 \\
195\end{array}$ & $\begin{array}{l}58 \\
78 \\
79\end{array}$ & $\begin{array}{l}62 \\
78 \\
68\end{array}$ \\
\hline 2 & $\begin{array}{l}\text { Basal } \\
\text { At } \\
\mathbf{P h}\end{array}$ & $\begin{array}{l}106 \\
114 \\
142\end{array}$ & $\begin{array}{l}110 \\
112 \\
144\end{array}$ & $\begin{array}{l}55 \\
95 \\
94\end{array}$ & $\begin{array}{l}60 \\
90 \\
90\end{array}$ \\
\hline 3 & $\begin{array}{l}\text { Basal } \\
\text { At } \\
\text { Ph }\end{array}$ & $\begin{array}{l}112 \\
125 \\
165\end{array}$ & $\begin{array}{l}128 \\
125 \\
165\end{array}$ & $\begin{array}{l}62 \\
99 \\
95\end{array}$ & $\begin{array}{l}63 \\
95 \\
82\end{array}$ \\
\hline 4 & $\begin{array}{l}\text { Basal } \\
\text { At } \\
\text { Ph }\end{array}$ & $\begin{array}{l}150 \\
145 \\
194\end{array}$ & $\begin{array}{l}150 \\
150 \\
194\end{array}$ & $\begin{array}{l}71 \\
87 \\
83\end{array}$ & $\begin{array}{l}70 \\
84 \\
82\end{array}$ \\
\hline 5 & $\begin{array}{l}\text { Basal } \\
\mathrm{At} \\
\mathrm{Ph}\end{array}$ & $\begin{array}{r}108 \\
98 \\
186\end{array}$ & $\begin{array}{l}134 \\
133 \\
182\end{array}$ & $\begin{array}{l}59 \\
66 \\
63\end{array}$ & $\begin{array}{l}61 \\
69 \\
63\end{array}$ \\
\hline 6 & $\begin{array}{l}\text { Basal } \\
\text { At } \\
\text { Ph }\end{array}$ & $\begin{array}{l}119 \\
110 \\
160\end{array}$ & $\begin{array}{l}122 \\
122 \\
161\end{array}$ & $\begin{array}{r}49 \\
102 \\
128\end{array}$ & $\begin{array}{r}47 \\
102 \\
112\end{array}$ \\
\hline 7 & $\begin{array}{l}\text { Basal } \\
\text { At } \\
\text { Ph }\end{array}$ & $\begin{array}{l}118 \\
118 \\
166\end{array}$ & $\begin{array}{l}127 \\
122 \\
166\end{array}$ & $\begin{array}{l}67 \\
71 \\
75\end{array}$ & $\begin{array}{l}61 \\
71 \\
75\end{array}$ \\
\hline 8 & $\begin{array}{l}\text { Basal } \\
\text { At } \\
\text { Ph }\end{array}$ & $\begin{array}{l}118 \\
123 \\
198\end{array}$ & $\begin{array}{l}128 \\
127 \\
206\end{array}$ & $\begin{array}{l}53 \\
77 \\
60\end{array}$ & $\begin{array}{l}50 \\
68 \\
57\end{array}$ \\
\hline 9 & $\begin{array}{l}\text { Basal } \\
\text { At } \\
\mathbf{P h}\end{array}$ & $\begin{array}{l}119 \\
119 \\
161\end{array}$ & $\begin{array}{l}116 \\
118 \\
161\end{array}$ & $\begin{array}{l}51 \\
82 \\
81\end{array}$ & $\begin{array}{l}65 \\
85 \\
82\end{array}$ \\
\hline 10 & $\begin{array}{l}\text { Basal } \\
\text { At } \\
\text { Ph }\end{array}$ & $\begin{array}{l}120 \\
124 \\
184\end{array}$ & $\begin{array}{l}138 \\
142 \\
185\end{array}$ & $\begin{array}{l}65 \\
98 \\
79\end{array}$ & $\begin{array}{r}67 \\
125 \\
99\end{array}$ \\
\hline \multicolumn{2}{|c|}{$\begin{array}{l}\text { Mean basal } \\
\text { SEM } \\
\text { P }\end{array}$} & $\begin{array}{r}123 \\
6 \\
<0.01\end{array}$ & $\begin{array}{r}131 \\
5\end{array}$ & $\begin{array}{l}59 \\
2 \\
\text { NS }\end{array}$ & $\begin{array}{r}61 \\
2\end{array}$ \\
\hline \multicolumn{2}{|c|}{$\begin{array}{l}\text { Mean At } \\
\text { SEM } \\
\mathbf{P}\end{array}$} & $\begin{array}{l}124^{\star} \\
6 \\
<0.02\end{array}$ & $\begin{array}{c}131^{\star} \\
5\end{array}$ & $\begin{array}{c}86 \neq \\
46 \\
\text { NS }\end{array}$ & $\begin{array}{c}87 \ddagger \\
6\end{array}$ \\
\hline \multicolumn{2}{|c|}{$\begin{array}{l}\text { Mean Ph } \\
\text { SEM } \\
\mathbf{P}\end{array}$} & $\begin{array}{c}175 \neq S \\
6 \\
\text { NS } \\
20\end{array}$ & $\begin{array}{c}176 \neq S \\
6\end{array}$ & $\begin{array}{c}83+\ddagger \\
6 \\
\text { NS }\end{array}$ & $\underset{5}{81 † \ddagger}$ \\
\hline
\end{tabular}

Basal, basal state; At, after atropine; $\mathrm{Ph}$, during pressure loading with phenylephrine; SEM, standard error of the mean; $P$, significance level; vs, versus; NS, not significant; * not significant vs basal state; + not significant vs atropine; $¥ \mathbf{P}<0.01$ vs basal state; $S \mathbf{P}<0.01$ vs atropine. increased to $1.39 \pm 0.08 \mathrm{diam} / \mathrm{s}$ after atropine, the results did not reach statistical significance. During the phenylephrine infusion the average mean VCF decreased to $1.17 \mathrm{diam} / \mathrm{s}(\mathrm{P}<0.01$ vs atropine, $\mathrm{NS}$ vs basal state). Placebo values were not significantly different from these.

Table 3 Effects of oral tocainide on left ventricular dimensions and ejection time during acute alterations in heart rate and blood pressure

\begin{tabular}{llll} 
Case State & $\frac{E D D(\mathrm{~cm})}{\text { no. }}$ & $\frac{\operatorname{ESD}(\mathrm{cm})}{\text { Placebo Tocainide }}$ & $\frac{\operatorname{LVET}(\mathrm{ms})}{\text { Placebo Tocainide }}$ \\
\hline Placebo Tocainide
\end{tabular}

\begin{tabular}{|c|c|c|c|c|c|c|c|}
\hline 1 & $\begin{array}{l}\text { Basal } \\
\text { At } \\
\text { Ph }\end{array}$ & $\begin{array}{l}6 \cdot 55 \\
6 \cdot 39 \\
6 \cdot 24\end{array}$ & $\begin{array}{l}6 \cdot 23 \\
6 \cdot 21 \\
6 \cdot 25\end{array}$ & $\begin{array}{l}3 \cdot 75 \\
3 \cdot 70 \\
3 \cdot 86\end{array}$ & $\begin{array}{l}3.55 \\
3.44 \\
3.83\end{array}$ & $\begin{array}{l}281 \\
249 \\
256\end{array}$ & $\begin{array}{l}277 \\
259 \\
269\end{array}$ \\
\hline 2 & $\begin{array}{l}\text { Basal } \\
\text { At } \\
\text { Ph }\end{array}$ & $\begin{array}{l}4 \cdot 87 \\
4 \cdot 94 \\
5 \cdot 24\end{array}$ & $\begin{array}{l}4 \cdot 96 \\
5 \cdot 05 \\
5 \cdot 18\end{array}$ & $\begin{array}{l}3.06 \\
3.47 \\
3.70\end{array}$ & $\begin{array}{l}3 \cdot 18 \\
3 \cdot 49 \\
3 \cdot 70\end{array}$ & $\begin{array}{l}334 \\
261 \\
299\end{array}$ & $\begin{array}{l}320 \\
267 \\
286\end{array}$ \\
\hline 3 & $\begin{array}{l}\text { Basal } \\
\text { At } \\
\text { Ph }\end{array}$ & $\begin{array}{l}4 \cdot 13 \\
3 \cdot 92 \\
4 \cdot 04\end{array}$ & $\begin{array}{l}4 \cdot 07 \\
3 \cdot 76 \\
4 \cdot 10\end{array}$ & $\begin{array}{l}2 \cdot 11 \\
2 \cdot 01 \\
2 \cdot 33\end{array}$ & $\begin{array}{l}2 \cdot 28 \\
2 \cdot 13 \\
2 \cdot 47\end{array}$ & $\begin{array}{l}330 \\
247 \\
285\end{array}$ & $\begin{array}{l}293 \\
242 \\
270\end{array}$ \\
\hline 4 & $\begin{array}{l}\text { Basal } \\
\text { At } \\
\text { Ph }\end{array}$ & $\begin{array}{l}7 \cdot 33 \\
7 \cdot 15 \\
7 \cdot 31\end{array}$ & $\begin{array}{l}7 \cdot 30 \\
7 \cdot 27 \\
7 \cdot 26\end{array}$ & $\begin{array}{l}4 \cdot 49 \\
4 \cdot 37 \\
4 \cdot 85\end{array}$ & $\begin{array}{l}4 \cdot 67 \\
4 \cdot 80 \\
4 \cdot 92\end{array}$ & $\begin{array}{l}319 \\
297 \\
334\end{array}$ & $\begin{array}{l}307 \\
288 \\
323\end{array}$ \\
\hline 5 & $\begin{array}{l}\text { Basal } \\
\text { At } \\
\text { Ph }\end{array}$ & $\begin{array}{l}4.85 \\
4.65 \\
5.03\end{array}$ & $\begin{array}{l}5 \cdot 02 \\
4 \cdot 88 \\
5 \cdot 16\end{array}$ & $\begin{array}{l}3.16 \\
2.97 \\
3.69\end{array}$ & $\begin{array}{l}3 \cdot 37 \\
3 \cdot 21 \\
3 \cdot 62\end{array}$ & $\begin{array}{l}293 \\
276 \\
325\end{array}$ & $\begin{array}{l}315 \\
294 \\
330\end{array}$ \\
\hline 6 & $\begin{array}{l}\text { Basal } \\
\text { At } \\
\text { Ph }\end{array}$ & $\begin{array}{l}5 \cdot 79 \\
4 \cdot 88 \\
4 \cdot 59\end{array}$ & $\begin{array}{l}5 \cdot 59 \\
4 \cdot 70 \\
4 \cdot 89\end{array}$ & $\begin{array}{l}3 \cdot 16 \\
2 \cdot 81 \\
2 \cdot 75\end{array}$ & $\begin{array}{l}3.23 \\
2.85 \\
3.03\end{array}$ & $\begin{array}{l}373 \\
267 \\
265\end{array}$ & $\begin{array}{l}380 \\
273 \\
285\end{array}$ \\
\hline 7 & $\begin{array}{l}\text { Basal } \\
\text { At } \\
\text { Ph }\end{array}$ & $\begin{array}{l}6 \cdot 14 \\
6 \cdot 10 \\
6 \cdot 18\end{array}$ & $\begin{array}{l}6 \cdot 24 \\
5 \cdot 97 \\
6 \cdot 20\end{array}$ & $\begin{array}{l}3 \cdot 19 \\
3 \cdot 37 \\
3 \cdot 19\end{array}$ & $\begin{array}{l}3 \cdot 20 \\
3 \cdot 48 \\
3 \cdot 28\end{array}$ & $\begin{array}{l}316 \\
308 \\
305\end{array}$ & $\begin{array}{l}312 \\
308 \\
297\end{array}$ \\
\hline 8 & $\begin{array}{l}\text { Basal } \\
\text { At } \\
\text { Ph }\end{array}$ & $\begin{array}{l}5 \cdot 48 \\
4.63 \\
5.55\end{array}$ & $\begin{array}{l}5 \cdot 70 \\
4 \cdot 89 \\
5 \cdot 47\end{array}$ & $\begin{array}{l}2 \cdot 87 \\
2 \cdot 82 \\
3 \cdot 58\end{array}$ & $\begin{array}{l}3 \cdot 28 \\
2 \cdot 99 \\
3 \cdot 67\end{array}$ & $\begin{array}{l}342 \\
273 \\
345\end{array}$ & $\begin{array}{l}351 \\
305 \\
368\end{array}$ \\
\hline 9 & $\begin{array}{l}\text { Basal } \\
\text { At } \\
\text { Ph }\end{array}$ & $\begin{array}{l}5.57 \\
5 \cdot 11 \\
5.56\end{array}$ & $\begin{array}{l}5 \cdot 70 \\
5 \cdot 66 \\
5 \cdot 79\end{array}$ & $\begin{array}{l}3.53 \\
3.33 \\
3.92\end{array}$ & $\begin{array}{l}3 \cdot 51 \\
3.58 \\
4 \cdot 08\end{array}$ & $\begin{array}{l}315 \\
281 \\
303\end{array}$ & $\begin{array}{l}302 \\
278 \\
301\end{array}$ \\
\hline 10 & $\begin{array}{l}\text { Basal } \\
\text { At } \\
\text { Ph }\end{array}$ & $\begin{array}{l}5 \cdot 69 \\
4 \cdot 70 \\
6 \cdot 00\end{array}$ & $\begin{array}{l}5 \cdot 67 \\
4 \cdot 71 \\
5 \cdot 95\end{array}$ & $\begin{array}{l}3.52 \\
3.05 \\
4.07\end{array}$ & $\begin{array}{l}3.45 \\
2.99 \\
3.93\end{array}$ & $\begin{array}{l}323 \\
264 \\
341\end{array}$ & $\begin{array}{l}310 \\
219 \\
293\end{array}$ \\
\hline \multicolumn{2}{|c|}{$\begin{array}{l}\text { Mean basal } \\
\text { SEM } \\
\mathbf{P}\end{array}$} & $\begin{array}{l}5 \cdot 64 \\
0.29\end{array}$ & $\begin{array}{l}5 \cdot 65 \\
0 \cdot 27 \\
\text { NS }\end{array}$ & $\begin{array}{l}3 \cdot 28 \\
0 \cdot 20\end{array}$ & $\begin{array}{l}3.37 \\
0.18 \\
\text { NS }\end{array}$ & $\begin{array}{r}323 \\
8\end{array}$ & $\begin{array}{r}317 \\
9 \\
\text { NS }\end{array}$ \\
\hline \multicolumn{2}{|c|}{$\begin{array}{l}\text { Mean At } \\
\text { SEM } \\
\mathbf{P}\end{array}$} & $\begin{array}{l}5 \cdot 24^{\star \star} \\
0 \cdot 31\end{array}$ & 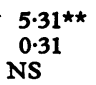 & $\begin{array}{l}3 \cdot 19 \star \\
0 \cdot 20\end{array}$ & $\begin{array}{l}3 \cdot 32^{\star} \\
0 \cdot 21 \\
\text { NS }\end{array}$ & $\begin{array}{c}272 \ddagger \\
6\end{array}$ & $\mathrm{NS}^{273 \ddagger}$ \\
\hline \multicolumn{2}{|c|}{$\begin{array}{l}\text { Mean Ph } \\
\text { SEM } \\
\mathbf{P}\end{array}$} & $\begin{array}{l}5 \cdot 57 * 9 \\
0 \cdot 29\end{array}$ & $\begin{array}{l}5 \cdot 63 *-\pi \\
0 \cdot 28 \\
\text { NS }\end{array}$ & \multicolumn{2}{|c|}{$\begin{array}{c}3.59^{\star \star \star} \int 3.65^{\star \star} S \\
0.220 .21 \\
\text { NS }\end{array}$} & $\begin{array}{c}306 * d \\
10\end{array}$ & $\begin{array}{c}302^{\star} \\
10 \\
N S\end{array}$ \\
\hline
\end{tabular}

Basal, basal state; At, after atropine; $\mathrm{Ph}$, during pressure loading with phenylephrine; SEM, standard error of the mean; $P$, significance level; vs, versus; NS, not significant; * not significant vs basal state; * $\mathbf{P}<0.05$ vs basal state; $¥ \mathbf{P}<0.01$ vs basal state; $\mathbf{P}<0.01$ ve atropine; $8 \mathrm{P}<0.05$ vs atropine; $\mathrm{EDD}$, end-diastolic dimension; ESD, end-systolic dimension; LVET, left ventricular ejection time. 
Table 4 Effects of oral tocainide on ultrasound measures of left ventricular performance in basal state and during acute alterations in heart rate and blood pressure

\begin{tabular}{|c|c|c|c|c|c|}
\hline \multirow{2}{*}{$\begin{array}{l}\text { Case } \\
\text { no. }\end{array}$} & \multirow[t]{2}{*}{ State } & \multicolumn{2}{|c|}{ Mean VCF $(\operatorname{diam} / \mathrm{s})$} & \multicolumn{2}{|c|}{ Mean VPW $\left(s^{-1}\right)$} \\
\hline & & Placebo & Tocainide & Placebo & Tocainide \\
\hline 1 & $\begin{array}{l}\text { Basal } \\
\text { At } \\
\mathbf{P h}\end{array}$ & $\begin{array}{l}1.52 \\
1.69 \\
1.49\end{array}$ & $\begin{array}{l}1.56 \\
1.72 \\
1.44\end{array}$ & $\begin{array}{l}1.01 \\
1.11 \\
0.96\end{array}$ & $\begin{array}{l}0.94 \\
1.12 \\
0.97\end{array}$ \\
\hline 2 & $\begin{array}{l}\text { Basal } \\
\text { At } \\
\mathbf{P h}\end{array}$ & $\begin{array}{l}1 \cdot 11 \\
1.14 \\
0.99\end{array}$ & $\begin{array}{l}1.12 \\
1.16 \\
1.00\end{array}$ & $\begin{array}{l}0.60 \\
0.66 \\
0.52\end{array}$ & $\begin{array}{l}0.64 \\
0.59 \\
0.62\end{array}$ \\
\hline 3 & $\begin{array}{l}\text { Basal } \\
\text { At } \\
\text { Ph }\end{array}$ & $\begin{array}{l}1.48 \\
1.97 \\
1.49\end{array}$ & $\begin{array}{l}1.40 \\
1.79 \\
1.47\end{array}$ & $\begin{array}{l}1.05 \\
1.37 \\
1.11\end{array}$ & $\begin{array}{l}1.04 \\
1.22 \\
1.04\end{array}$ \\
\hline 4 & $\begin{array}{l}\text { Basal } \\
\mathrm{At} \\
\mathbf{P h}\end{array}$ & $\begin{array}{l}1.22 \\
1.31 \\
1.01\end{array}$ & $\begin{array}{l}1 \cdot 17 \\
1.18 \\
1.00\end{array}$ & $\begin{array}{l}0.71 \\
0.76 \\
0.58\end{array}$ & $\begin{array}{l}0.68 \\
0.71 \\
0.56\end{array}$ \\
\hline 5 & $\begin{array}{l}\text { Basal } \\
\text { At } \\
\text { Ph }\end{array}$ & $\begin{array}{l}1 \cdot 19 \\
1.31 \\
0.82\end{array}$ & $\begin{array}{l}1 \cdot 16 \\
1 \cdot 16 \\
0.90\end{array}$ & $\begin{array}{l}0.94 \\
0.99 \\
0.58\end{array}$ & $\begin{array}{l}0.91 \\
0.96 \\
0.66\end{array}$ \\
\hline 6 & $\begin{array}{l}\text { Basal } \\
\text { At } \\
\mathbf{P h}\end{array}$ & $\begin{array}{l}1.22 \\
1.59 \\
1.51\end{array}$ & $\begin{array}{l}1.11 \\
1.44 \\
1.33\end{array}$ & $\begin{array}{l}0.67 \\
0.78 \\
0.88\end{array}$ & $\begin{array}{l}0.60 \\
0.62 \\
0.71\end{array}$ \\
\hline 7 & $\begin{array}{l}\text { Basal } \\
\text { At } \\
\mathrm{Ph}\end{array}$ & $\begin{array}{l}1.52 \\
1.45 \\
1.59\end{array}$ & $\begin{array}{l}1.56 \\
1.35 \\
1.59\end{array}$ & $\begin{array}{l}0.81 \\
0.77 \\
0.76\end{array}$ & $\begin{array}{l}0.64 \\
0.88 \\
0.78\end{array}$ \\
\hline 8 & $\begin{array}{l}\text { Basal } \\
\text { At } \\
\mathbf{P h}\end{array}$ & $\begin{array}{l}1.39 \\
1.43 \\
1.03\end{array}$ & $\begin{array}{l}1.21 \\
1.27 \\
0.89\end{array}$ & $\begin{array}{l}0.72 \\
0.88 \\
0.57\end{array}$ & $\begin{array}{l}0.65 \\
0.88 \\
0.58\end{array}$ \\
\hline 9 & $\begin{array}{l}\text { Basal } \\
\text { At } \\
\text { Ph }\end{array}$ & $\begin{array}{l}1 \cdot 16 \\
1.24 \\
0.97\end{array}$ & $\begin{array}{l}1.27 \\
1.32 \\
0.98\end{array}$ & $\begin{array}{l}0.75 \\
0.88 \\
0.59\end{array}$ & $\begin{array}{l}0.68 \\
0.76 \\
0.56\end{array}$ \\
\hline 10 & $\begin{array}{l}\text { Basal } \\
\text { At } \\
\text { Ph }\end{array}$ & $\begin{array}{l}1 \cdot 18 \\
1.33 \\
0.94\end{array}$ & $\begin{array}{l}1 \cdot 26 \\
1 \cdot 67 \\
1 \cdot 16\end{array}$ & $\begin{array}{l}0.74 \\
0.91 \\
0.49\end{array}$ & $\begin{array}{l}0.74 \\
1 \cdot 10 \\
0.63\end{array}$ \\
\hline \multicolumn{2}{|c|}{$\begin{array}{l}\text { Mean basal } \\
\text { SEM } \\
\text { P }\end{array}$} & $\begin{array}{l}1.30 \\
0.05\end{array}$ & $\begin{array}{l}1.29 \\
0.06 \\
\text { NS }\end{array}$ & $\begin{array}{l}0.80 \\
0.05\end{array}$ & $\begin{array}{l}0.75 \\
0.05 \\
\text { NS }\end{array}$ \\
\hline \multicolumn{2}{|c|}{$\begin{array}{l}\text { Mean At } \\
\text { SEM } \\
\mathbf{P}\end{array}$} & $\begin{array}{l}1.44^{\star} \\
0.08\end{array}$ & $\begin{array}{l}1.39 \star \\
0.08 \\
\text { NS }\end{array}$ & $\begin{array}{l}0.91 \star \star \\
0.07\end{array}$ & $\begin{array}{l}0.87 \star \star \\
0.07 \\
\text { NS }\end{array}$ \\
\hline \multicolumn{2}{|c|}{$\begin{array}{l}\text { Mean Ph } \\
\text { SEM } \\
\mathbf{P}\end{array}$} & $\begin{array}{l}1 \cdot 17 \star S \\
0.10\end{array}$ & $\begin{array}{l}1.17 \star S \\
0.09 \\
\text { NS }\end{array}$ & $\begin{array}{l}0.70 \star S \\
0.07\end{array}$ & $\begin{array}{l}0.71 \text { *S } \\
0.05 \\
\text { NS }\end{array}$ \\
\hline
\end{tabular}

Basal, basal state; $\mathrm{At}$, after atropine; $\mathrm{Ph}$, during pressure loading with phenylephrine; SEM, standard error of the mean; $P$, significance level; vs, versus; NS, not significant; * not significant vs basal state; $\star \star P<0.05$ vs basal state; $\int P<0.01$ vs atropine. Mean VCF, mean velocity of circumferential fibre shortening; mean VPW, mean normalised posterior wall velocity.

The average mean VPW during tocainide administration was $0.75 \pm 0.05 \mathrm{~s}^{-1}$. This increased to $0.87 \pm 0.07 \mathrm{~s}^{-1}$ after atropine $(P<0.05)$, and then decreased to $0.71 \pm 0.05 \mathrm{~s}^{-1}(P<0.01$ vs atropine, NS vs basal state) during phenylephrine infusion. Placebo values did not differ significantly from these values.
During the increase in heart rate after atropine, the mean left ventricular end-diastolic dimension in the placebo and tocainide groups decreased (both $P<0.05$ vs basal state), and returned to basal state levels during phenylephrine infusion. The mean left ventricular end-systolic dimension did not change significantly in either group after atropine, but increased in both groups during phenylephrine infusion $(P<0.01$ vs atropine, $P<0.05$ vs basal state). In both the placebo and tocainide groups the mean left ventricular ejection times shortened after atropine $(P<0.01$ vs basal state), and then returned towards basal state values during phenylephrine infusion ( $P<0.05$ vs atropine, NS vs basal state). Thus, for the placebo and tocainide groups alike, the increases in mean VCF values after atropine were largely the result of reductions in the left ventricular ejection time, and the decreases in mean VCF values during phenylephrine infusion were largely the result of increases in end-systolic dimensions and a return towards basal levels of left ventricular ejection times.

A typical response to an acute increase in heart rate and systemic arterial pressure is shown in the echocardiograms in the Fig. Though these recordings. were made during tocainide therapy the response was the same whether the patient was taking tocainide or placebo.

\section{Discussion}

Little information is available concerning the effects of tocainide on left ventricular function. Duce et al. (1973), in a study of 6 conscious dogs, reported that oral tocainide in a dose of $25-200 \mathrm{mg} / \mathrm{kg}$ every 1 to 4 hours produced no alterations in systemic arterial or central venous pressure. However, Coltart et al. (1974) found moderate depression of cardiac function in 4 anaesthetised dogs given single oral tocainide doses of $120 \mathrm{mg} / \mathrm{kg}$. Significant increases in left ventricular end-diastolic pressure and significant decreases in heart rate and left ventricular $\mathrm{dP} / \mathrm{dt}$ were observed; plasma tocainide concentrations in both studies ranged from 15 to $30 \mu \mathrm{g} / \mathrm{ml}$.

There are 5 previous reports of preliminary data concerning the use of tocainide as an antiarrhythmic agent in humans and in no study was cardiac depression observed (McDevitt et al., 1976; Winkle et al., 1976; Woosley et al., 1977; Ryan et al., 1978; Winkle et al., 1978). However, the doses of tocainide employed were, per $\mathrm{kg}$ body weight, less than onetenth the dose used by Coltart et al. (1974) in their animal study. Further, in none of these studies was the response to any controlled stress, such as acute increases in systemic arterial pressure, evaluated. In the present study the mean plasma drug concentration in the tocainide group at the time of the echo- 
cardiographic studies was $5.3 \mu \mathrm{g} / \mathrm{ml}$, the range being from $2 \cdot 0$ to $7 \cdot 3 \mu \mathrm{g} / \mathrm{m}$.

Nine of 10 patients had levels within the lower limits of the usual therapeutic range $(>4 \mu \mathrm{g} / \mathrm{ml})$. These therapeutic levels were the result of choosing a dose of $400 \mathrm{mg}$ of tocainide every 8 hours. This dose level was selected as a safety precaution because at the outset of the study the potential for myocardial depression of tocainide was unknown.

When mean VCF and mean VPW values are used to compare the inotropic status of the myocardium in an individual on different occasions as was done in this study it is important that heart rates and blood pressures are matched (Hirshleifer et al., 1975). In the basal state the mean heart rates were similar in the tocainide and placebo groups but the mean systemic systolic blood pressures were slightly higher in the tocainide group. Experimental studies (Ross et al., 1966; Mahler et al., 1975) and studies in humans (Hirshleifer et al., 1975; Quinones et al., 1975) have documented a reduction in the extent and velocity of myocardial shortening as resistance to ejection is increased. However, despite the increase in mean basal state systolic blood pressure during tocainide, no significant reduction in mean VCF or mean VPW occurred. After atropine the mean heart rates for the placebo and tocainide groups did not differ significantly from each other. However, just as in the basal state, the mean systolic arterial pressure was higher in the tocainide group than in the placebo group. Again no significant differences were found between the mean VCF and mean VPW values in both groups.

The effects of acute alterations in systemic arterial pressure on isovolumic and ejection phase indices of left ventricular performance have previously been studied in our laboratory (Mahler et al., 1975). This same technique has also been used to study the effects of drugs on left ventricular function (Mahler et al., 1974; Crawford et al., 1975; Gorwit et al., 1975; Crawford et al., 1976). In both normal conscious dogs (Mahler et al., 1974) and normal human subjects (Crawford et al., 1976) oral digoxin had a protective effect on left ventricular performance during acute pressure loading. In these studies the positive inotropic effect of digoxin was more apparent during than before acute pressure loading. By contrast, using similar techniques, we have been able to show that beta-adrenergic blockade exerts a modest but definite depressant effect on left ventricular function (LeWinter et al., 1978). Further, another antiarrhythmic agent, quinidine gluconate, can be shown to be a myocardial depressant during acute afterloading when sympathetic tone is suppressed by beta-adrenergic blockade (Engler et al., 1978). Therefore, we reasoned that if tocainide is a myocardial depressant, a further reduction in left ventricular performance compared with placebo values should be demonstrable during acute pressure loading. However, no latent alteration in left ventricular performance due to tocainide was 'unmasked'-the average mean VCF and mean VPW values during phenylephrine infusion were similar whether the patients were receiving tocainide or placebo.

Thus, under the conditions of our study tocainide in a typical therapeutic dose of $\mathbf{4 0 0} \mathrm{mg}$ each $\mathbf{8}$ hours had no myocardial depressant action. However, no patient had severely depressed left ventricular function in the basal state (partly because patients with coronary artery disease and congestive cardiomyopathy were specifically excluded). The effect of tocainide on ventricular function when severely depressed function is present at rest or when higher drug doses are used is, therefore, still unknown. Thus the effects of tocainide on cardiac performance resemble those of lignocaine, which in large doses in experimental animals produces myocardial depression (Austen and Moran, 1965; Nayler et al., 1969; Asokan et al., 1968) but in conventional doses in man is devoid of significant cardiovascular effects (Harrison et al., 1963; Grossman et al., 1969; Rahimtoola et al., 1971). Tocainide, however, has a major advantage that lignocaine does not have: it can be administered orally.

In summary, tocainide is an oral antiarrhythmic agent with no evident myocardial depressant effect when typical therapeutic drug doses are used in patients without severe left ventricular dysfunction. If the reported efficacy of tocainide is further confirmed, and if no severe toxic problems result from chronic administration of the drug, then it ought to be a useful antiarrhythmic agent. In this short-term study no laboratory abnormalities occurred during tocainide administration, but further long-term testing is required to demonstrate that the drug has no adverse systemic effects.

We are grateful to $\mathrm{Mr}$ Richard Braun for technical assistance, and Mrs Elizabeth Gilpin for statistical advice.

\section{References}

Asokan, S. K., Frank, M. H., and Regan, T. J. (1968). Assessment of myocardial depression with therapeutic doses of lidocaine and procainamide (abstract). American fournal of Cardiology, 21, 91.

Austen, W. G., and Moran, J. M. (1965). Cardiac and peripheral vascular effects of lidocaine and procainamide. American fournal of Cardiology, 16, 701-707.

Coltart, D. J., Berndt, T. B., Kernoff, R., and Harrison, D. C. (1974). Antiarrhythmic and circulatory effects of Astra 
W36095, a new lidocaine-like agent. American fournal of Cardiology, 34, 35-41.

Crawford, M. H., Karliner, J. S., and O'Rourke, R. A. (1976). Favorable effects of oral maintenance digoxin therapy on left ventricular performance in normal subjects: echocardiographic study. American fournal of Cardiology, 38, 843-847.

Crawford, M. H., O'Rourke, R. A., Garza, G., and Karliner, J. S. (1975). Oral propranolol: effects on left ventricular function in normal subjects (abstract). Circulation, 51 and 52, Suppl. II, 172.

Duce, B. R., Smith, E. R., Boyes, R. N., and Byrnes, E. W. (1973). The acute antiarrhythmic and toxic effects of 2-amino-2',6'-proprionoxylidide (W36095) in mice and dogs (abstract). Pharmacologist, 15, 192.

Engler, R. L., LeWinter, M., Van Nyhuish, L., Kaiser, R., and Karliner, J. (1978). Depressant effects of quinidine gluconate on left ventricular contraction and relaxation in conscious dogs with volume overload (abstract). American Fournal of Cardiology, 41, 416.

Gorwit, J., Crawford, M., Karliner, J., and O'Rourke, R. (1975). Echocardiographic assessment of left ventricular performance in normal subjects receiving oral quinidine (abstract). Circulation, 51 and 52, Suppl. II, 19.

Grossman, J. I., Cooper, J. A., and Frieden, J. (1969). Cardiovascular effects of infusion of lidocaine on patients with heart disease. American fournal of Cardiology, 24, 191-197.

Harrison, D. C., Sprouse, J. H., and Morrow, A. G. (1963). The antiarrhythmic properties of lidocaine and procaine amide. Circulation, 28, 486-491.

Hirshleifer, J., Crawford, M., O'Rourke, R. A., and Karliner, J. S. (1975). Influence of acute alterations in heart rate and systemic arterial pressure on echocardiographic measures of left ventricular performance in normal human subjects. Circulation, 52, 835-841.

LeWinter, M. M., Karliner, J. S., and O'Rourke, R. A. (1978). Effects of oral equiblocking doses of a cardioselective and noncardioselective $\beta$-adrenergic blocking agent on left ventricular function in the normal conscious dog. Cardiovascular Research, 12, 81-90.

McDevitt, D. G., Nies, A. S., Wilkinson, G. R., Smith, R. F., Woosley, R. L., and Oates, J. A. (1976). Antiarrhythmic effects of a lidocaine congener, tocainide, 2-amino-2',6'propionoxylidide, in man. Clinical Pharmacology and Therapeutics, 19, 396-402.

Mahler, F., Karliner, J. S., and O'Rourke, R. A. (1974). Effects of chronic digoxin administration on left ventricular performance in the normal conscious dog. Circulation, 50, 720-727.

Mahler, F., Ross, J., Jr., O'Rourke, R. A., and Covell, J. W. (1975). Effects of changes in preload, afterload and inotropic state on ejection and isovolumic phase measures of left ventricular contractility in the conscious dog. American Fournal of Cardiology, 35, 626-634.

Nayler, W. G., McInnes, I., Carson, V., Stone, J., and Lowe, T. E. (1969). The effect of lignocaine on myocardial function, high energy phosphate stores and oxygen consumption: a comparison with propranolol. American Heart Fournal, 78, 338-345.

Popp, R. L., Filly, K., Brown, O. R., and Harrison, D. C. (1975). Effect of transducer placement on echocardiographic measurements of left ventricular dimensions. American fournal of Cardiology, 35, 537-540.

Quinones, M. A., Gaasch, W. H., Cole, J. S., and Alexander, J. K. (1975). Echocardiographic determination of left ventricular stress-velocity relations in man, with reference to the effects of loading and contractility. Circulation, 51, 689-700.

Rahimtoola, S. H., Sinno, M. Z., Loeb, H. S., Chuquimia, R., Rosen, K. M., and Gunnar, R. M. (1971). Lidocaine infusion in acute myocardial infarction. Archives of Internal Medicine, 128, 416-418.

Ross, J., Jr., Covell, J. W., Sonnenblick, E. H., and Braunwald, E. (1966). Contractile state of the heart characterized by force-velocity relations in variability afterloaded and isovolumic beats. Circulation Research, 18, 149-163.

Ryan, W., Engler, R., LeWinter, M., Bloomquist, J., and Karliner, J. S. (1978). Treatment of refractory ventricular arrhythmias with tocainide: preliminary report. In Proceedings of a Symposium on Acute and Long Term Management of Myocardial Ischaemia, Copenhagen, 8-9 September, 1977. Ed. by $\AA$. Hjalmarson and L. Wilhelmsen. Astra Pharmaceuticals AB, Stockholm.

Winer, B. J. (1971). Statistical Principles in Experimental Design, pp. 514-603. McGraw-Hill, New York.

Winkle, R. A., Meffin, P. J., Fitzgerald, J. W., and Harrison, D. C. (1976). Clinical efficacy and pharmacokinetics of a new orally effective antiarrhythmic, tocainide. Circulation, 54, 884-889.

Winkle, R. A., Meffin, P. J., and Harrison, D. C. (1978). Long-term tocainide therapy for ventricular arrhythmias. Circulation, 57, 1008-1016.

Woosley, R. L., McDevitt, D. G., Nies, A. S., Smith, R. F., Wilkinson, G. R., and Oates, J. A. (1977). Suppression of ventricular ectopic depolarizations by tocainide. Circulation, $56,980-984$.

Requests for reprints to $\mathrm{Dr}$ Joel S. Karliner, University of California Medical Center, 225 Dickinson Street, San Diego, California 92103, USA. 